\title{
Clinical Aspects
}

\section{Antiphospholipid antibodies - selected topics}

\author{
Ronald A. Asherson
}

Lupus Research Unit, St. Thomas' Hospital, London SEl 7EH, UK.

\section{The 'primary' antiphospholipid syndrome}

As many of the clinical and serological manifestations associated with the antiphospholipid antibodies will be covered by other speakers and because of the limited time at my disposal, a brief overview will be given of some of our recent work in this field. These areas include:

1. Valve lesions and the association of valve and cerebral pathology

2. Myocardial infarction and antiphospholipid antibodies

3. The 'primary' antiphospholipid syndrome

\section{Valve lesions}

Since the report by Chatash in 1986 documenting 11 patients with antiphospholipid antibodies, severe dysfunctional valve lesions (aortic and mitral incompetence), accompanied by arterial occlusions affecting particularly the cerebral vasculature tree, there has been a surge of interest and publications on the apparent increased frequency of valve lesions in the subset of patients with the antiphospholipid syndrome (APS). However, other recent publications have drawn attention to the frequency of valve lesions, including valve 'thickening' in the general lupus population and it is now clear that the use of Doppler in addition to $2 \mathrm{M}$ echocardiography, will demonstrate that a much higher number of lupus patients in fact show valve abnormalities than had previously been detected. The original description by Libman and Sacks of small non-infective vegetations seen at postmortem in lupus patients has been considerably expanded. Large haemodynamically significant valve lesions affecting both the mitral and aortic valves, causing incompetence and rarely stenosis, are being increasingly frequently reported. These may be so severe as to warrant valve replacement. There is no evidence at this time that there is any increase in frequency of valve lesions in patients with APS. However, what is now appreciated is that thrombosis may form on damaged valves as well as in the heart chambers themselves, and may give rise to thromboembolic complications. These affect the cerebral vessels particularly, causing transient ischaemic att- acks and strokes and, rarely, embolism may result to other organs, and affect the coronary vessels, spleen, kidneys, and lungs (from right-sided thrombi).

What is unclear is whether these thrombi may be formed on the 'thickened valves' which are undetectable clinically and doubtless this question will be answered in time. Diagnostic difficulties may arise in lupus patients who develop vegetations on damaged valves, which are accompanied by fever and evidence of lupus activity clinically and serologically, as evidence shows in two recently illustrative cases.

Infective endocarditis is more frequently seen in lupus patients, but as in the 'culture negative' patient with the above features, anticoagulation therapy should be instituted as the risk of thromboembolic complications may be high. Our patients were treated successfully with antibiotic cover, prednisolone in high dosage, as well as warfarin with good results. Studies on sera from patients with infective endocarditis, show that although positive latex tests are not uncommon, significant anticardiolipin antibody elevations do not occur. Borderline low positive levels (IgM) are the rule. Thrombus may also occur on prosthetic valves and result in obstruction and death. Several reports of this complication in patients with the lupus anticoagulant have been published.

\section{Myocardial infarction (MI)}

Coronary vessel occlusion is much less frequently encountered than cerebrovascular disease. We have studied 13 patients seen over the past 4 years most of whom have been $<30$ years of age. In two, the MI was silent, detected on routine electrocardiography. In 9/13 the MI was preceded by years of recurrent deep venous thrombosis and pulmonary thromboembolism. In two, the acute events occurred after the discontinuation of warfarin therapy and in one, was associated with oral contraceptive usage. An additional 3 patients with coronary ischaemic symptoms were encountered. In two, the onset of angina pectoris was associated with poor warfarin control. One patient had manifested warfarin 'resistance'. 


\section{The primary antiphospholipid syndrome}

Since the early publications in the 1960 s and 1970s on the 'lupus anticoagulant' it has been evident that almost $50 \%$ of patients with antiphospholipid antibodies do not suffer from classical lupus as defined by the 1982 revised criteria for the classification of SLE. Some of these patients have been described as having 'lupus-like disease' or 'atypical lupus' when they have 3 or less of these criteria present, as well as other 'autoimmune' features not included in these criteria (such as Sjögren's and Raynaud's). A second group has now been defined - the 'primary' antiphospholipid syndrome. These patients are often male, with low positive antinuclear antibodies (usually $<1: 160$ ) and the absence of other autoantibodies such as antibodies to double stranded DNA (ds DNA) and extractable nuclear antigens (ENAs). They may however have Coombs' positivity or frank haemolytic anaemia, sometimes accompanied by thrombocytopenia (Evans' syndrome), antibodies to mitochondria and false positive VDRL. There may be a family history of SLE or 'autoimmune disease' elicitable, demonstrating the close relationship to the 'lupus syndrome'. Several patients initially presenting with elevated DNA binding, and other features such as discoid LE have seemingly entered this category without ever develop-

\section{References}

1. Asherson, R.A. \& Lubbe, W.F. Cerebral valve lesions in SLE associated with antiphospholipid antibodies (Editorial). J Rheumatol 1988, 15: 539-543.

2. Asherson, R.A., Gibson, D.G., Evans, W.E. et al. Diagnostic and therapeutic problems in two patients with antiphospholipid antibodies, heart valve-lesions and transient ischaemia. (Abstract). Ann Rheum Dis 1988, 47: 947-953. ing any other features of lupus, while others, (a minority) followed over years, have developed typical SLE. The majority, however, seem to remain in the category of 'primary' antiphospholipid syndrome. We have examined the features of 70 of these patients drawn from several centres. Almost $50 \%$ have had recurrent venous thrombosis, particularly deep venous thrombosis, often accompanied by pulmonary thromboembolism. Two patients had Budd-Chiari syndrome. Transient ischaemic attacks and strokes have been evident in one third and other arterial occlusions have also been encountered-myocardial infarctions, ileo-femoral, mesenteric, renal, digital vessel ischaemia (causing gangrene of toes). Recurrent fetal loss was present in $\mathbf{4 0} \%$. Thrombocytopenia, Coombs' positivity and haemolytic anaemia were less common. Two patients had pulmonary hypertension, one thromboembolic, one resembling the primary non-thromboembolic type. One had nodular skin lesions and two suffered from vasculitic skin rashes. Two patients had a history of chorea. Only one patient was found to have a valve lesion (A1) perhaps discounting the seemingly high frequency of valve lesions with antiphospholipid antibodies rather linking uncomplicated valve lesions with the underlying lupus process itself.

3. Asherson, R.A., Khamashta, M.A., Gil, A. et al. Cerebrovascular disease and antiphospholipid antibodies in systemic lupus erythematosus, lupus like disease and the primary antiphospholipid syndrome. Am J Med 1989, 86: $391-399$. 